\title{
Etude comparée de l'efficacité de la cyperméthrine et deux bioinsecticides, Beauveria bassiana et suneem contre l'altise du gombo, Podagrica spp (Coleoptera: Chrysomelidae)
}

\author{
Agbéko Kodjo TOUNOU ${ }^{1 *}$, Komi AGBOKA ${ }^{1}$, Batossa Emmanuel BAKOUMA ${ }^{1}$, \\ Mewezenon AADOM ${ }^{1}$, Anani Kossi Mawuko ADJEVI ${ }^{1}$ et Komla SANDA ${ }^{1,2}$ \\ ${ }^{I}$ Laboratoire de Recherche sur les Sciences des Agroressources et Santé Environnementale (LARASE), \\ Ecole Supérieure d'Agronomie (ESA) de l'Université de Lomé, Togo. \\ ${ }^{2}$ Université de Kara, Togo. \\ *Auteur correspondant, E-mail: ktounou@gmail.com
}

\section{RESUME}

Parmi les ravageurs du gombo, Abelmoschus Spp L., les altises, Podagrica Spp (Coleoptera: Chrysomelidae), causent d'importants dommages à la culture. Les fortes infestations de l'insecte réduisent les activités photosynthétiques de la plante, entraînant des pertes élevées de rendement. La présente étude vise à comparer l'effet de deux bioinsecticides, Suneem 1\% EC, 10 g/l d'azadirachine (20\%), Beauveria bassiana (1 $\mathrm{x} 10^{11}$ spores/ha) et le cyperméthrine 50 EC (1 $1 /$ ha), un insecticide de synthèse, pour le contrôle de Podagrica Spp, en conditions de champ. Deux applications des différentes formulations ont été faites à intervalle de 6 jours sur la partie aérienne des plantes. Les résultats ont montré que la cyperméthrine a causé la plus forte réduction de la densité de population des altises $(89,56 \pm 0,76)$, suivi de Suneem $(81,24 \pm 2,40)$ et du $B$. bassiana $(64,90 \pm 3,07)$. De même le pourcentage des feuilles attaquées et le pourcentage de surface foliaire endommagée par l'insecte ont été significativement affectés par les traitements. Il ressort de cette étude que $B$. Bassiana et le Suneem, peuvent être utilisés pour lutter efficacement contre les populations d'altises de gombo, Podagrica Spp en condition de champ.

(C) 2018 International Formulae Group. All rights reserved.

Mots clés: Bioinsecticide, champignon entomopathogène, altise du gombo, réduction de la densité.

\section{Comparative study of the efficacy of cypermethrin and two bioinsecticides, Beauveria bassiana and Suneem against the flea beetle, Podagrica spp (Coleoptera: Chrysomelidae) on okra crops}

\begin{abstract}
The flea beetles, Podagrica Spp (Coleoptera: Chrysomelidae), is among the most important pests that cause significant economic damage to okra, Abelmoschus spp L. Heavy infestations of the insect reduces the photosynthetic activities of the plant, resulting in high losses of yield. The present study aimed at comparing the effect of two bioinsecticides, Suneem $1 \%$ EC, $10 \mathrm{~g} / 1$ azadirachin (20\%), Beauveria bassiana $\left(1 \times 10^{11}\right.$ spores / ha) and cypermethrin 50 EC (1 / ha), a synthetic insecticide, for the control of Podagrica Spp, under
\end{abstract}


field conditions. Two applications of the different formulations were made at 6-day intervals on the aerial part of the plants. The results showed that cypermethrin caused the greatest reduction in the population density of flea beetles $(89.56 \pm 0.76)$, followed by Suneem $(81.24 \pm 2.40)$ and B. bassiana $(64,90 \pm 3.07)$. Percent of leaf attack and leaf area damage by the insect were significantly lowered by the B. bassiana and Suneem application compared to the untreated control. It appears from this study that B. Bassiana and Suneem could be used to effectively control of okra flea beetles populations under field conditions.

(C) 2018 International Formulae Group. All rights reserved.

Keywords: Bioinsecticide, entomopathogenic fungus, okra flea beetle, density reduction.

\section{INTRODUCTION}

Le gombo, Abelmochus esculentus (L.) Moench (Malvales : Malvacea), est un légume originaire d'Afrique. Le gombo joue un rôle important dans l'alimentation humaine (Kahlon et al., 2007; Khomsug et al., 2010) en fournissant des graisses, protéines, hydrates de carbone, phosphore, calcium, fer, soufre, fibre, minéraux et vitamines (Gopalan et al., 2007). La plante est cultivée dans les régions tropicales, subtropicales et méditerranéennes pour sa forte valeur marchande, sa richesse en éléments nutritif et son importance dans l'alimentation des populations des villes et des campagnes. C'est une importante culture végétale valorisée pour ses feuilles et fruits immatures comestibles comme soupe et sauce (Khomsug et al., 2010) dans la plupart des pays d'Afrique subsaharienne, Le fruit contient de nombreux éléments nutritifs (calcium, fer, glucides, protéines, vitamines, etc.) qui sont des compléments alimentaires nécessaires à l'alimentation de base constituée principalement d'amidon (céréales et tubercules). Selon Siemonsma et Kouamé 2(004), 100 grammes de gombo contiendraient 7,5 g de glucides, 5,76 g hydrate de carbone, $2 \mathrm{~g}$ de fibres, $1,52 \mathrm{~g}$ de protéines, $1,7 \mathrm{~g}$ de lipides, 256,6 $\mathrm{mg}$ de potassium, 50,4 mg de Calcium, $46 \mathrm{mg}$ de magnésium, 13,04 $\mathrm{mg}$ de vitamine $\mathrm{C}, 0,4 \mathrm{mg}$ de Fer, 36,5 micro grammes d'acides foliques. Outre les apports nutritionnels, les grains, les infusions du fruit, la décoction du fruit immature, des feuilles et fleurs ainsi que les extraits des feuilles et les infusions des racines ont des propriétés pharmacologiques et utilisés en médecine traditionnelle pour le traitement du diabète et des bronchites (Sabitha et al., 2011; Roy et al., 2014).

Au Togo comme dans la plus part des pays de l'Afrique de l'Ouest, la production du gombo est d'une importance capitale surtout sur le plan économique et joue un rôle essentiel dans l'équilibre nutritionnel des populations rurales. Selon le rapport du recensement agricole de 2012 sur la production national du gombo au Togo était de 1278,66 tonnes pour une superficie de 117,64 ha (FAO, 2013). L'importance économique du gombo, ses diverses utilisations et son importance dans le régime alimentaire des populations font de cette culture, une denrée importante de la sécurité alimentaire et nutritionnelle et de la réduction de la pauvreté. La production du gombo se trouve cependant entraver par des facteurs tels que les conditions climatiques, la non maitrise des techniques culturales, la pauvreté des sols, les maladies et les insectes ravageurs qui limitent sa production et son rendement. Les insectes ravageurs affectent négativement non seulement le rendement du gombo mais aussi déprécient la qualité des fruits récoltés. Les altises, Podagrica Spp (Coleoptera : Chrysomelidae), ont été reconnues comme étant des insectes les plus dangereux et les plus nuisibles de la culture du gombo (Fondio et al., 2007a ; Soro et al., 2016). Cet insecte attaque surtout les feuilles en y faisant des trous qui lui sont très caractéristiques. La défoliation provoquée par cet insecte entraîne une baisse de la photosynthèse et par conséquent réduit le rendement de la production. Des études ont montré que cet insecte est responsable de la transmission du Virus de la Mosaïque du Gombo (OMV) observé au Nigeria, en Côte d'ivoire, au 
Kenya et en Sierra Leone (Ugwoke et Onyishi, 2009). Les pertes occasionnées par ces divers insectes ravageurs justifient la mise en place de plusieurs moyens de lutte parmi lesquels la lutte chimique reste la plus ancienne et la plus utilisée. En effet, des études ont montré que l'utilisation des insecticides serait profitable vu des dégâts énormes surtout au point de vue économique qu'infligeraient les insectes comme $P$. uniformis sans les traitements insecticides. Cependant, les problèmes de pollution de l'eau et des sols associés à l'utilisation de pesticides chimiques de synthèse, l'apparition du phénomène de résistance ainsi que l'interférence négative de ces pesticides sur la santé humaine, sur l'environnement et la pression constante qu'exerce le consommateur pour une agriculture plus saine ont poussé à l'intensification des recherches pour la mise au point de méthodes alternatives plus respectueuses de l'environnement. Par ailleurs, le coût élevé des insecticides chimiques de synthèse les rend inaccessibles aux paysans africains et appellent à la recherche de solutions alternatives pour limiter l'attaque des insectes. Des biopesticides à base de plantes à effet insecticides comme le neem (Nguema Ndoutoumou et al., 2015 ; Mondedji et al., 2016) et des champignons entomopathogènes dont Beauveria bassiana sont de plus en plus utilisés dans les luttes contre les ravageurs des cultures (Godonou et al., 2009; James et al., 2009).

B. bassiana (Bals.) Vuill. (Deuteromycotina: Hyphomycetes) dont la pathogénicité a été démontrée la première fois par Bassi en 1835, représente à ce jour le champignon entomopathogène le plus largement utilisé à travers le monde (Godonou et al., 2009; Douro Kpindou et al., 2012). B. bassiana a été rapporté sur une large gamme d'insectes et est capable infecter aussi bien les stades immatures et les adultes des (Cherry et Nursely, 2010 ; Douro Kpindou et al., 2012). C'est un important agent pathogène commercialisé et utilisé comme un agent de lutte biologique au champ pour la gestion des insectes ravageurs d'importance agricole
(Godonou et al., 2009; James et al., 2009 ). Ce champignon a un grand potentiel en tant que agent de control biologique du fait aussi bien de la facilité de sa production en masse et de sa formulation que pour sa spécificité d'action (Zurek et al., 2000) et pourra donc constituer un alternatif au produits chimiques dans la production du gombo. La présente étude a été réalisée pour évaluer l'efficacité comparée de B. bassiana, du neem et de la cyperméthrine sur les altises du gombo en conditions du champ et au laboratoire.

\section{MATERIEL ET METHODES Site de l'étude}

Cette étude a été conduite à la Station d'Expérimentation Agronomique de Lomé $\left(06^{\circ} 17^{\prime} \mathrm{N}, 001^{\circ} 21^{\prime} \mathrm{E}\right)$, et au Laboratoire de Recherche sur les Agroressources et Santé Environnementale (LARASE) de l'Ecole Supérieure d'Agronomie de l'Université de Lomé pendant la grande saison de pluie de 2012. Le site d'essais est localisé dans la région maritime du Togo, caractérisée par deux saisons de pluies avec le maximum en juin et septembre, et une pluviométrie annuelle moyenne de $1200 \mathrm{~mm}$.

\section{Provenance des semences du gombo}

Les semences de gombo variété locale, une variété à fruits côtelés gluants, provenant d'Afagnan, localité situés à $80 \mathrm{~km}$ de la ville de Lomé, ont été utilisées pour leur préférence par les producteurs du milieu.

\section{Préparation des suspensions}

L'objectif de la présente étude était de déterminer l'efficacité de trois produits de traitement, un produit chimique de synthèse, la cyperméthrine, et deux biopesticides, le champignon $B$. bassiana et le suneem sur l'abondance des populations d'altises de gombo et évaluer leurs effets sur les dégâts causés aux feuilles de gombo. La souche Bb11 de B. bassiana initialement isolée de Helicoverpa armigera (Hübner) (Lepidoptera : Noctuidae) et testée pour sa pathogénicité par Douro Kpindou et al. (2012), a été obtenue de l'unité de production de l'IITA 
Bénin. Le taux moyen de germination des conidies utilisés dans les essais calculé en suivant la méthode décrite par Tounou et al. (2003) est de 94\%. Le Suneem 1\% EC, un insecticide végétal extrait des graines du neem (Azadirachta indica, A. Juss) et dont la matière active, l'azadirachine $(10 \mathrm{~g} / \mathrm{l})$, principal composé du neem, a été appliqué à la dose de $20 \%$ selon les recommandations du projet PEDUNE/PRONAF. L'insecticide de synthèse, la cyperméthrine $50 \mathrm{EC}$ a été quant à lui appliqué à la dose recommandée de de 11/ha (Fondio et al. (2007b).

Pour les tests d'efficacité au champ, les suspensions des différents produits à base d'eau ont été testées contre les altises adultes. Pour préparer la suspension de champignon, $10 \mathrm{~g}$ de poudre de conidie de B. bassiana ont été mis en suspension dans 11 d'eau potable (équivalent à $10^{11}$ spores/l, déterminée sur la base des essais préliminaires) pour faciliter l'adhésion des conidies aux feuilles lors du traitement et éviter leur dessèchement, $1,5 \mathrm{ml}$ d'huile d'arachide a été ajoutée à la suspension.

\section{Dispositif expérimental et application des formulations au champ}

Les essais ont été mis en place au champ suivant un dispositif en blocs aléatoires constitués de quatre traitements répétés chacun quatre fois. Les parcelles mesuraient $6 \times 5 \mathrm{~m}$ et les blocs consécutifs sont séparés de $2 \mathrm{~m}$. Les allées ont été semées de maïs deux semaines avant le semi du gombo pour réduire les interactions entre les différents traitements. Deux graines de gombo ont été semées par poquets suivant un schéma de $0,5 \mathrm{~m} \mathrm{x} 1 \mathrm{~m}$ et le démariage à un plant par poquet a été fait 2 semaines après semis. Les traitements ont débuté au $19^{\text {ème }}$ jour après semis. Les traitements sont appliqués deux fois par intervalle de six jours et les observations trois jours après chaque traitement (Ekra, 2010) à l'aide d'un pulvérisateur manuel de 21 (Pressure sprayer). Toutes les applications ont été réalisées le matin et à chaque fois la plante entière est pulvérisée. L'eau potable utilisée pour la dilution des produits de traitement a servi de traitement témoin.

\section{Evaluation de la densité des altises}

Les densités des altises, déterminées sur la base de la densité du ravageur par plant ont été évaluées au jour 0 (jour de traitement), et après à trois jour d'intervalle jusqu'au jour 18 après traitement pour avoir l'effet des traitements et le niveau de population du ravageur avant le traitement suivant. Les insectes ont été comptés en inspectant rigoureusement les deux faces des feuilles de 40 plants choisis de façon aléatoire par traitement en raison de 10 plants choisis au hasard sur les 4 lignes centrales de chaque parcelle.

\section{Evaluation des dégâts sur les feuilles du gombo}

Le nombre de feuilles attaquées et la défoliation des feuilles de gombo en condition d'infestation naturelle par les altises de gombo ont été évalués sur 10 plants choisis au hasard sur les 4 lignes centrales de chaque parcelle au jour de la première application (jour 0) et à un intervalle de 3 jours jusqu'au jour 18, en utilisant la méthode décrite par Dumont (2014) pour la défoliation du soja. Le nombre de feuilles attaquées et la défoliation ont été estimés en termes du pourcentage de feuilles présentant des signes d'attaque des altises et du pourcentage de la surface foliaire détruite par le ravageur. Le nombre de feuilles attaquées et la proportion de surface foliaire endommagée après application des produits ont été cumulés du jour 3 au jour 18 .

\section{Analyse statistique des données}

L'efficacité des différents traitements a été utilisée pour tracer les histogrammes et comparer les différents traitements entre eux. Les données ont été soumises à une analyse de la variance après transformation par le logarithme $(\log (X+1)$ et Arcsine $\sqrt{ }($ percent $\mathrm{x} / 100$ ), respectivement pour les densités d'altise et les pourcentages de feuilles 
attaquées ainsi que le pourcentage de la surface foliaire endommagée. Lorsque l'ANOVA a révélé des différences significatives entre traitements, le test SNK (Student-Newman Keuls), au seuil de 5\%, a été fait afin de séparer les moyennes des différents traitements. Pour toutes ces analyses, le logiciel SPSS version 20 a été utilisé. Sachant que la densité d'une population d'insectes traités dépend de la taille de la population initiale au sein des parcelles et la progression des fluctuations non seulement liées aux traitements mais aussi aux changements naturels de la population, nous avons calculé la valeur corrigée du pourcentage de l'efficacité de chaque traitement en utilisant la formule de Henderson-Tilton :

$\%$ Efficacité $=\left[1-\left(\frac{T a}{T b}\right) \times\left(\frac{C b}{C a}\right)\right] \times 100$, où $T_{b}$ et $C_{b}$ représentent la densité avant le traitement dans les parcelles traitées et les parcelles témoin, respectivement, $T_{a}$ et $C_{a}$ représentent les densités après traitement dans les parcelles traitées et les parcelles témoin, respectivement (Henderson et Tilton, 1955). Les densités après chaque traitement ont été cumulées du jour 3 au jour 18. Les données ont été analysées par ANOVA et les moyennes sont discernées par le test de Student-Newman-Keuls au seuil de $\alpha=0.05$.

\section{RESULTATS}

\section{Evolution de la population d'altises après traitement}

Le nombre moyen d'altises par plant compté sur les parcelles traitées et non traitées durant les 18 jours d'observation est présenté dans la Figure 1. Sur les parcelles non traitées, le nombre moyen d'altises par plant a augmenté et est passé de 9,37 $\pm 0,74$ à 12,7 \pm 1,53 insecte. Les densités dans les parcelles traitées sont cependant réduites de 9,90 $\pm 1,27$ à $1,6 \pm 0,3$, de $9,43 \pm 1,03$ à $1,40 \pm 0,3$; et de $7,47 \pm 1,26$ à $0,90 \pm 0,2$, respectivement dans les parcelles traitées avec du B. bassiana,
Suneem et la cyperméthrine. Des différences significatives ont été observées entre les différents traitements $\left(\mathrm{Df}_{3 ; 6}=13,86, \mathrm{P}<\right.$ 0.0001 ), avec la plus faible densité enregistrée dans le traitement à la Cyperméthrine, suivi de Suneem et de $B$. bassiana. Les plus fortes densités ont été enregistrées dans le traitement témoin (Figure 1).

L'efficacité des différents traitements exprimée en pourcentage de réduction de la population d'altises est présentée dans le Tableau 1. L'efficacité des traitements diffère significativement avec le traitement à la Cyperméthrine entraînant la meilleure réduction de la population d'altises suivie de Suneem et de B. bassiana.

\section{Evaluation des dégâts sur les feuilles du gombo}

La double application du produit chimique de synthèse et des deux produits biologiques ont permis une protection des plants de gombo contre les attaques des altises réduisant ainsi les dommages causés aux feuilles comparée au traitement témoin (Tableau 2). Durant les 18 jours d'évaluation, des différences significatives ont été observées aussi bien au niveau des pourcentages de feuilles attaquées et du pourcentage de la surface foliaire endommagée entre les parcelles traitées et les parcelles non traitées (Tableau 2). Comparativement aux parcelles témoins, les dommages associés au comportement alimentaire du ravageur (attaque des feuilles et surface foliaire défoliée), sont significativement faibles dans les parcelles traitées. Les résultats ont montré que lorsque les plants de gombo ont reçu des traitements insecticides à base de la cyperméthrine et de suneem, aucune différence statistique n'est obtenue aussi bien en termes de feuilles attaquées et de surface foliaire endommagée (Tableau 2). 


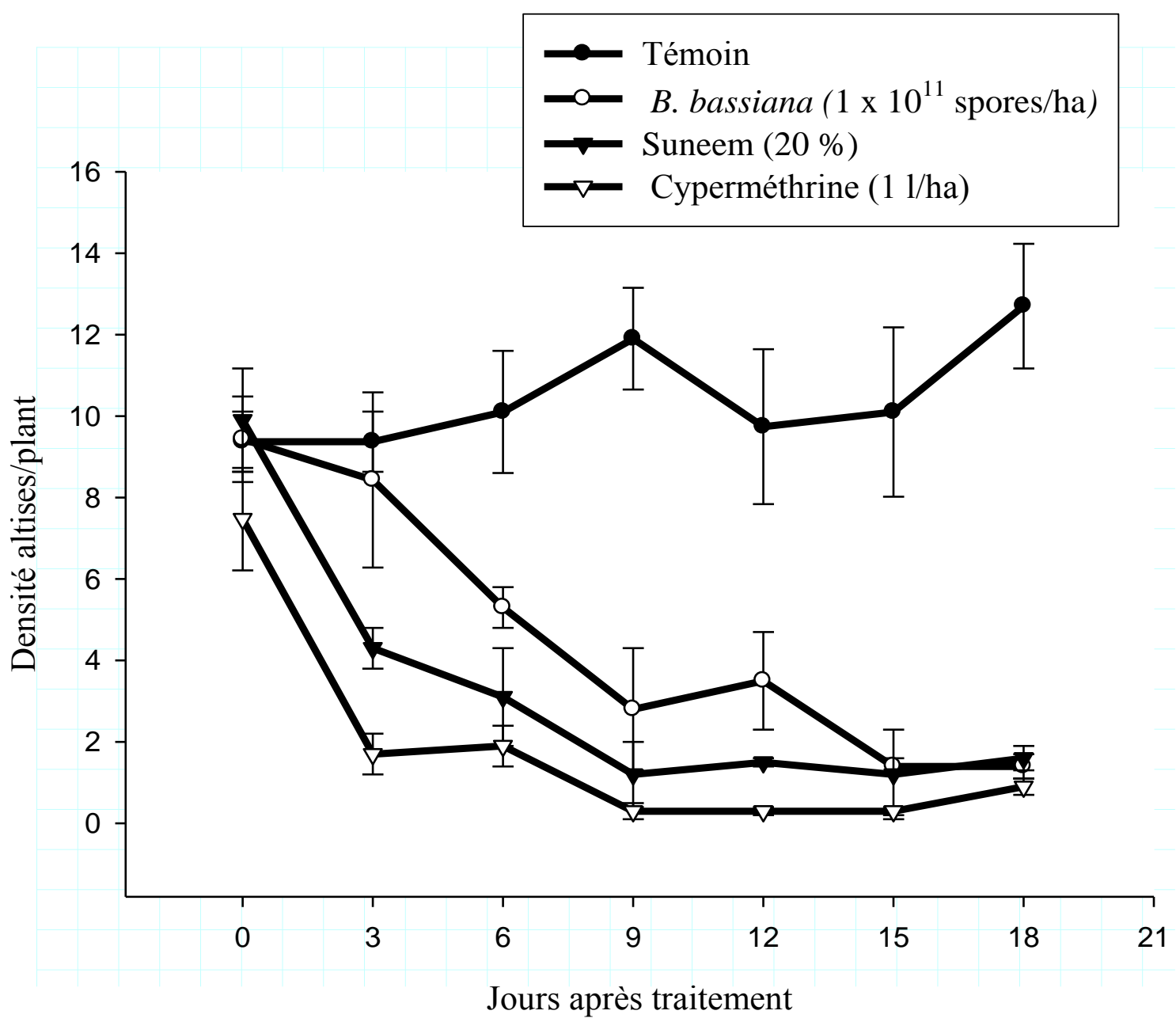

Figure 1: Nombre moyen ( \pm ES) des altises adultes par plant de gombo.

Tableau 1: Efficacités des différents traitements calculées sur la base de la densité le jour des applications pendant les 18 jours d'évaluation en utilisant la formule de Henderson et Tilton (1955).

\begin{tabular}{ll}
\hline Traitements $^{\mathfrak{\varepsilon}}$ & Réduction de la de la densité des altises $(\% \pm \mathbf{E S})^{\boldsymbol{\beta}}$ \\
\hline Cyperméthrine (11/ha) & $89,56 \pm 0,76 \mathrm{a}$ \\
Suneem (20\%) & $81,24 \pm 2,40 \mathrm{~b}$ \\
B. bassiana (1x 1011 spores/ha) & $64,90 \pm 3,07 \mathrm{c}$ \\
df & $2 ; 6$ \\
F & 19,67 \\
P & 0,0001 \\
\hline
\end{tabular}

${ }^{\beta}$ Les moyennes suivies de différentes lettres sont significativement différentes ( $\mathrm{P}<0,05$, test de Student-Newman-Keuls). ${ }^{\mathfrak{f}}$ Les densités après traitements ont été cumulées du jour 3 au jour 18. 
Tableau 2: Défoliation et pourcentage de feuilles attaquées par les attises sur les plants de gombo traités à la cyperméthrine, Suneem et B. bassiana.

\begin{tabular}{|c|c|c|}
\hline Traitements $^{\beta}$ & Feuilles attaquées $\left((\% \pm \mathrm{ES})^{\mathfrak{f}}\right.$ & surface foliaire endommagée $((\% \pm \mathrm{ES})$ \\
\hline Témoin $^{\mathrm{a}}$ & $98,86 \pm 0,01 \mathrm{a}$ & $75,29 \pm 1.02 \mathrm{a}$ \\
\hline $\begin{array}{l}\text { B. bassiana }\left(1 \times 10^{11}\right. \\
\text { spores/ha })\end{array}$ & $89,71 \pm 0,07 b$ & $59,02 \pm 0.95 b$ \\
\hline Cypermethrine (11/ha) & $83,24 \pm 2,40 c$ & $40,78 \pm 1,07 \mathrm{c}$ \\
\hline Suneem $(20 \%)$ & $79,56 \pm 0,76 c$ & $38,41 \pm 2,09 \mathrm{c}$ \\
\hline df & $3 ; 6$ & $3 ; 6$ \\
\hline $\mathrm{F}$ & 17,37 & 12,26 \\
\hline $\mathrm{P}$ & 0,0001 & 0,0001 \\
\hline
\end{tabular}

${ }^{\beta}$ Le nombre de feuilles attaquées et taux de défoliation après traitements ont été cumulées du jour 3 au jour 18.

${ }^{f}$ Les moyennes suivies de différentes lettres sont significativement différentes ( $\mathrm{P}<0,05$, test de Student-Newman-Keuls).

\section{DISCUSSION}

Les résultats de la présente étude ont montré que par rapport au témoin non traité, les différentes formulations insecticides ont effectivement réduit la population des altises en condition du champ. Le produit chimique de synthèse (Cyperméthrine) s'est montré significativement plus efficace dans la réduction de la population d'altises sur les plants de gombo, suivi du Suneem et du $B$. bassiana. Ceci serait dû à la différence de pouvoir insecticide et du mode d'action des différentes formulations d'une part, et à la différence de la rémanence des différents produits dans le champ. D'une manière générale, les produits chimiques de synthèse ont un pouvoir insecticides plus élevé et reste actifs pour un temps relativement plus long après application comparés aux produits biologiques (Tounou et al., 2012) lesquels sont caractérisés par une biodégradabilité sous l'effet du soleil.

La réduction de la population d'altise dans les parcelles traitées au Suneem serait en partie lié aux effets insecticides, mais beaucoup plus aux effets répulsifs et antinutritionnels des produits à base de neem qu'aux effets de mortalité directe. Ceci se confirme bien par le faible taux d'attaque des feuilles et le faible niveau de défoliation de la surface foliaire relativement observés au champ. Les produits à base de neem inhibent en effet la prise de nourriture et la croissance des insectes appartenant à différents d'insectes (Nathan et al., 2007; Schenk et al., 2001; Mondedji et al., 2014). Nos résultats sont en accord avec ceux de Gnago et al. (2010) qui ont rapporté que les extraits de graines de neem réduisaient la densité des populations $d$ es altises du gombo. Ce résultat est toute fois en contradiction avec celui rapporté par Ekra (2010) qui a montré dans ses essais que l'azadirachtine ne pouvait pas assurer un contrôle efficace des altises. Les résultats des travaux de Schenk et al. (2001) ont montré que lorsque les composantes du neem spécialement l'azadirachtine entre dans le corps des larves, l'activité de l'ecdysone (enzyme contrôlant le passage du stade larvaire au stade adulte) est supprimée. Ceci empêche la métamorphose chez ces dernières qui demeurent à l'état larvaire jusqu'à la mort. Il est à noter également que les propriétés répulsives et antiapétant du neem auraient joué aussi un rôle non négligeable dans la réduction de la densité des altises sur les plants de gombo traité au Suneem. Le nombre réduit d'insectes par plant, le faible pourcentage des feuilles attaquées et le niveau réduit de la défoliation des feuilles obtenus dans cette étude sur les parcelles traitées au Suneem, pourraient s'expliquer par l'effet répulsif et antiapétant du suneem, un produit à base du neem.

La souche Bb11 du B. bassiana utilisée dans cette étude a montré une virulence élevée 
aussi bien en condition de champ qu'au laboratoire et possèderait ainsi un bon potentiel pour la lutte biologique contre les altises du gombo, $P$ uniformis. La mortalité relativement élevée observée au laboratoire confirme bien la virulence des champignons entomopathogènes en général (Tounou et al., 2003) et de la souche Bb11 de B. bassiana en particulier pour la lutte contre les insectes ravageurs des cultures (Douro Kpindou et al., 2012). Sur les larves de Maruca vitrata (Lepidoptera: Crambidae), Mehinto et al. (2014) ont rapporté des mortalités allant de 30 à $100 \%$ en 10 jours d'incubation des larves inoculées aux spores de B. bassiana et de Metarhizium anisopliae. Des résultats similaires ont été rapportés par Douro Kpindou et al. (2012) qui ont montré une forte virulence de la souche Bb11 à contrôler sur les larves de H. armigera. Sur le doryphore de la pomme de terre, Leptinotarsa decemlineata Say (Coleoptera : Chrysomelidae) et le puceron vert du pêcher, Myzus persicae (Sulzer) (Homoptera : Aphididae), Todorova et al. (2000) ont rapporté de forte virulence de différents isolats de $B$. bassiana

\section{Conclusion}

Bien que plusieurs champignons pathogènes sont connus comme agents pathogènes dans divers ordre d'insectes notamment les coléoptères, à ce jour le potentiel des entomopathogènes à contrôler les altises du gombo n'a pas été évaluée. Cette étude a le mérite de montrer la susceptibilité des altises du gombo à la souche Bb11 disponible et dont l'efficacité a déjà été confirmée contre différents insectes d'importance économique comme $P$. xylostella, $\mathrm{H}$. armigera et Bemisia argentifolii (Godonou et al., 2009 ; Douro Kpindou et al., 2012). Des études additionnelles sont toutefois nécessaires pour déterminer l'effet de différentes doses aussi bien de B. bassiana que du Suneem sur le ravageur. Par ailleurs, les effets des conditions environnementales sur la persistance des deux produits biologiques doivent aussi être étudiés pour une évaluation complète $\mathrm{du}$ potentiel biopesticide du Suneem et $B$. bassiana à contrôler les altises du gombo.

\section{CONFLITS D'INTERETS}

Les auteurs déclarent n'avoir aucun conflit d'intérêts.

\section{REMERCIEMENTS}

Les auteurs tiennent à remercier les autorités de l'Institut International d'Agriculture Tropicale (IITA) pour les spores de Beauveria bassiana et les techniciens de Laboratoire de Recherche sur les Sciences des Agroressources et Santé Environnementale pour les appuis apportés dans la réalisation de cette étude.

\section{RÉFÉRENCES}

Cherry R, Nuessly G. 2010. Repellency of the biopesticide, Azadirachtin, to wireworms (Coleoptera: Elateridae). Florida Entomologist., 93: 52-55. DOI: https://doi.org/10.1653/024.093.0107

Douro Kpindou OK, Djegui DA, Glitho IA, Tamò M 2012. Réponse des stades larvaires de Helicoverpa armigera (Hübner) (Lepidoptera: Noctuidae) à l'application de champignons entomopathogènes Metarhizium anisopliae et Beauveria bassiana. Biotechnologie, Agronomie, Société et Environement, 16: 283-293.

Dumont A. 2014. Défoliation dans le soja.https://www.lebulletin.com/actualite s/defoliation-dans-le-soya-66572 consulté le 20 septembre 2017.

Ekra KA. 2010. Etude comparée de l'efficacité des extraits aqueux de graines de neem (Azadirachta indica Juss) et de feuilles d'eucalyptus (Eucalyptus camaldulensis) dans la lutte contre les insectes du gombo (Abelmoschus esculentus L). Mémoire de fin de cycle pour l'obtention du diplôme d'Ingénieur en agriculture générale, Institut national polytechnique Félix Houphouët- Boigny, 78p.

FAO. 2013. Aperçu de l'agriculture togolaise à travers le pré-recensement 2012, Ministère de l'Agriculture et de la pêche, Secrétariat Général, 122p. 
Fondio L, Kouamé C, Djidji AH, Traoré D. 2007a. Caractérisation des systèmes de culture intégrant le gombo dans le maraîchage urbain et périurbain de Bouaké dans le Centre de la Côte d'Ivoire. International Journal of Biological and Chemical Sciences, 5: 1178-1189.

DOI: http://dx.doi.org/10.4314/ijbcs.v5i3.7225 1

Fondio L, Djidji A H, Kouamé C, Aïdara S, Hala N 2007b. Bien cultiver le gombo en Côte d'Ivoire. CNRA-CTA, 4p.

Gnago JA, Danho M, Atcham Agneroh T, Fofana IK, , Kohou AG 2010. Efficacité des extraits de neem (Azadirachta indica) et de papayer (Carica papaya) dans la lutte contre les insectes ravageurs $\mathrm{du}$ gombo (Abelmosehus esculentus) et $\mathrm{du}$ chou (Brassica oleracea) en Côte d'Ivoire. International Journal of Biological and Chemical Sciences, 4: 953-966.

DOI: http://dx.doi.org/10.4314/ijbcs.v4i4.6303 5

Godonou I, James B, Atcha-Ahowe C, Vodouhè S, Kooyman C, Ahanchédé A, Korie, S 2009. Potential of Beauveria bassiana and Metarhizium anisopliae isolates from Benin to control Plutella xylostella L. (Lepidoptera: Plutellidae). Crop Protection, 28: 220224.

DOI: https://doi.org/10.1016/j.cropro.2008.10. 009.

Gopalan C, Sastri SBV, Balasubramanian S. 2007. Nutritive value of Indian foods, National Institute of Nutrition (NIN), ICMR, India. Haraprasad N, Nirandjana SR, Prakash HS, Shetty HS et Wahab S 2001. Beauveria bassiana a potential mycopesticide for the efficient control of coffee berry borer, Hypothenemus hampei (Ferrari) in India. Biocontrol Sciences and Technology, 11: 251-260. DOI:

https://doi.org/10.1080/09583150120035 675.

James B, Godonou I, Atcha-Ahowe C. 2009. Promoting biopesticide candidates from experimental to commercial level for sustainable vegetable production. Pesticide Management in West Africa, 7: 34-52.

DOI: http://hdl.handle.net/10568/90251.

Kahlon TS, Chapman MH, Smith GE. 2007. In vitro binding of bile acids by okra beets asparagus eggplant turnips green beans carrots and cauliflower. Food Chemistry, 103: 676-680. DOI: 10.1016/j.foodchem.2006.07.056.

Khomsug P, Thongjaroenbuangam W, Pakdeenarong N, Suttajit M, Chantiratikul P 2010. Antioxidative activities and phenolic content of extracts from Okra (Abelmoschus esculentus L.) Research Journal of Biological Sciences, 5: 310-13.

Mehinto JT, Atachi P, Douro Kpindou OK, Dannon EA, Tamò M 2014. Mortality of Maruca vitrata (Lepidoptera: Crambidae) larval stages induced by different doses of the entomopathogenic fungi Metarhizium anisopliae and Beauveria bassiana. International Journal of Advanced Research, 2: 273285.

Mondedji AD, Kasseney BD, Nyamador WS, Abbévi Abbey G, Amevoin K, Adéoti R, Ketoh KG, GLITHO IA 2016. Effets d'extrait hydroéthanolique de feuilles de neem (Azadirachta indica A. Juss) sur Plutella xylostella (Lepidoptera : Plutellidae) et Lipaphis erysimi (Hemiptera : Aphididae) dans la production du chou au Sud du Togo. International Journal of Biological and Chemical Sciences, 10: 1666-1677. DOI: http://dx.doi.org/10.4314/ijbcs.v8i5.30.

Mondedji AD, Nyamador WS, Amevoin K, Ketoh GK, Glitho IA. 2014. Efficacité d'extraits de feuilles de neem Azadirachta indica (Sapindale) sur Plutella xylostella (Lepidoptera : Plutellidae), Hellula undalis (Lepidoptera : Pyralidae) et Lipaphis erysimi (Hemiptera : Aphididae) du chou Brassica oleracea (Brassicaceae) dans une approche « Champ Ecole Paysan» au sud du Togo. International Journal of Biological and Chemical Sciences, 8: 2286-2295. 10.4314/ijbcs.v8i5.30. 
Nathan SS, Choi MY, Chae HP, Seo HY, Jae DK, Seok MK 2007. The toxic effects of neem extract and azadirachtin on the brown planthopper, Nilaparvata lugens (Stål) (BPH) (Homoptera: Delphacidae). Chemosphere, 67: 80-88. DOI: 10.1016/j.chemosphere.2006.09.045.

Nguema Ndoutoumou P, Ondo Ovono P, Gatarasi T, Olery Okoumba JS 2015. Effet des extraits de Jatropha curcas L. et de Tabernanthe iboga Baill. dans la lutte contre le scolyte du caféier (Hypothenemus hampei Ferrari) au SudEst du Gabon. International Journal of Biological and Chemical Sciences 9: 2764-2775.

DOI: http://dx.doi.org/10.4314/ijbcs.v9i6.20 .

Roy A, Shrivastava SL, Mandal SM 2014. Functional properties of Okra Abelmoschus esculentus L. (Moench): traditional claims and scientific evidences. Plant Science Today, 1: 121130.

DOI: http://dx.doi.org/10.14719/pst.2014.1.3.6 3.

Sabitha V, Ramachandran S, Naveen KR, Panneerselvam K 2011. Antidiabetic and antihyperlipidemic potential of Abelmoschus esculentus (L.) Moench. In streptozotocin-induced diabetic rats. Journal of Pharmacy and Bioallied Sciences, 3: 397-402. DOI: 10.4103/0975-7406.84447

Schenk P, Imdorf A, Fluri P 2001. Effets de l'huile de neem sur l'acarien varroa et les abeilles. Revue Suisse d'Apiculture, 98 : 114-119.

Siemonsma JS, Kouamé C. 2004. Abelmoschus esculentus (L) Moench. In Ressources végétales de l'Afrique Tropicale, tome 2: Légumes, Grubben GJH, Denton OA (Eds). pp2530.

Soro S, Yéboué NL, Tra Bi CS, Zadou DA, Koné I 2016. Dynamics of the flea beetle Podagrica decolorata Duvivier, 1892 (Insecta: Chrysomelidae) on okra crops: implications for conservation of the Tanoe-Ehy Swamp Forests (southeastern Ivory Coast). Journal of Animal \&Plant Sciences, 30: 4758-4766.
Todorova SI, Coderre1 D, Côté J-C. 2000. Pathogenicity of Beauveria bassiana isolates toward Leptinotarsa decemlineata (Coleoptera: Chrysomelidae), Myzus persicae (Homoptera: Aphididae) and their predator Coleomegilla maculata lengi (Coleoptera: Coccinellidae). Phytoprotection, 81: 15-22. DOI: 10.7202/706196ar.

Tounou AK, Sokame B M, Akpavi S, Ganyo KK, Ketoh, KG, Gumedzoe YMD 2012. Effets des extraits végétaux sur la dynamique de populations des insectes ravageurs de niébé, Vigna unguiculata Walp, dans le sud du Togo. Journal de la Recherche Scientifique de l'Université de Lomé (Togo), Série A, 14: 25-34.

Tounou AK, Agboka K, Poehling HM, Raupach K, Langewald J, Zimmermann G, Borgemeister C. 2003. Evaluation of the Entomopathogenic Fungi Metarhizium anisopliae and Paecilomyces fumosoroseus (Deuteromycotina: Hyphomycetes) for Control of the Green Leafhopper Empoasca decipiens (Homoptera: Cicadellidae) and Potential Side Effects on the Egg Parasitoid Anagrus atomus (Hymenoptera: Mymaridae). Biocontrol Science and Technology, 13: 715-728. DOI:

https://doi.org/10.1080/09583150310001 606534.

Ugwoke KI, Onyishi LE. 2009. Effects of Mycorrhizae (Glomus musae), Poultry Manure and Okra Mosaic Potyvirus (Okmv) on Yield of Okra (Abomoscus esculentus). Production Agriculture and Technology, 5: 359-369.

Zurek L, Keddie BA. 2000. Beauveria bassiana (Balsamo) Vuilemin a promising microbial control agent of the satin moth (Lepidoptera: Lymanthriidae). Biocontrol Sciences and Technology, 10: 641-644. DOI: https://doi.org/10.1080/09583150075001 6433. 